\title{
CFD ANALYSIS OF CAR BODY AERODYNAMICS INCLUDING EFFECT OF PASSIVE FLOW DEVICES - A REVIEW
}

\author{
Asif Ahmed ${ }^{1}$, Murtaza M A ${ }^{2}$ \\ ${ }^{I}$ Research Scholar, Automobile Engineering, Amity University (Lucknow Campus),Uttar Pradesh, India \\ ${ }^{2}$ Professor, ASET, Amity University (Lucknow Campus),Uttar Pradesh, India
}

\begin{abstract}
With the emphasis lying on increasing fuel efficiency of vehicles in order to combat rising fuel prices and environmental challenges the manufacturers are thinking beyond the conventional vehicle systems by focusing on its aerodynamics. Aerodynamic drag exceeds 50 per cent of the total resistance to motion at speeds above $70 \mathrm{~km} / \mathrm{hr}$, and above $100 \mathrm{~km} / \mathrm{hr}$ it is the most important factor. The review is done to identify the various shortcomings of the automotive designers when it is in regards to flow separation of air at the rear of the vehicle which causes most of the losses. This paper focuses on the work already done in the field of aerodynamics starting with Ahmed Body. It is a bluff body with adjustable rear slant angle and the basis upon which the aerodynamicists test their models. And then, moving onto passive aerodynamic enhancements for automobiles like vortex generators and diffusers whose various dimensional modulations were discussed with several steps leading to its advancement in vehicle body design. This brings to the simulation, Computational Fluid Dynamics (CFD) and its role in this analysis was covered. CFD has been modified a lot from the beginning to increase the accuracy of its predictions. So the paper lists various simulation techniques studied by the previous researchers in order to understand the wake region behind the car which has been notoriously difficult to predict till date. Several aspects of aerodynamic drag that need further analysis to improve the aerodynamic were highlighted.
\end{abstract}

Keywords: Drag Force, Drag Coefficient, Ahmed Body, CFD Simulation, Vehicle Aerodynamics, Passive Flow Devices

\section{INTRODUCTION}

Automotive industry is the second most researched engineering field in the world after aerospace. Cars used to be very crude and unrefined but with the passage of time the manufacturers had to device methods to refine the vehicle in order to improve its working condition as well as to improve its performance efficiencies. The obvious first choice was to start from the engine. And after initial high performance gains the design of these engines and its components are nearing its maximum potential and the gains are not as much as it used to be earlier. So the manufacturers are veering into different areas to gain more performance. They now design every component of every system of the vehicle to work at its highest efficiency while being as lightweight as possible. Another area where manufacturers are interested to work upon is the aerodynamics of it. With massive gains in performance and handling characteristics of the vehicle as shown by the race cars over the years with aerodynamic devices attached to them, commercial vehicle manufacturers are now bucking the trend and more interested to incorporate a more aerodynamic design to their vehicles.

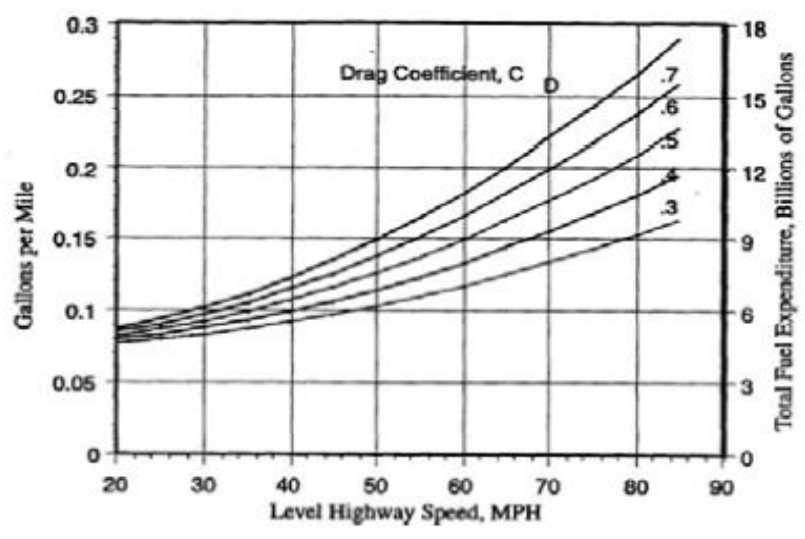

Fig-1: Fuel economy with reduction in drag coefficient [4]

Fig-1 depicts the fuel economy offered by the car at various speeds and how it varies in accordance with the drag coefficient. According to Hucho [1] in a medium-sized European car, aerodynamic drag accounts for nearly $80 \%$ of total road resistance at $100 \mathrm{kmph}$. So it is very binding on the designers to focus on decreasing this drag by implementing design changes at the rear and also by the addition of the passive control devices. Krishnani [2] had described the fuel usage of cars in both urban and highway driving. The vehicle shape uses about $3 \%$ of fuel to overcome the resistance it offers in urban driving whereas it takes $11 \%$ of fuel in case of highway driving. And this considerable 
increase of fuel usage at cruising speeds is what attracts several automotive research centers to automotive aerodynamics. This led to optimization of car aerodynamics more precisely the reduction of the drag coefficient $\left(C_{d}\right)$ which is mostly characterized by the outer shape of the vehicle [3].

The automotive research centers across the globe then started designing cars that were more slippery in the air which would give minimum drag coefficient $\left(\mathrm{C}_{\mathrm{d}}\right)$. Earlier the $C_{D}$ values ranged from 0.7-0.65 for the old box shaped car profiles. But now it has come down to around 0.3 for the recent streamlined bodies [5]. But aerodynamics has always remained a very expensive proposition and wasn't entertained by the small and medium car manufacturing industries.And to solve it analytically was near to impossible because, the turbulence created behind the vehicle was where most of the power losses occurred and it was impossible to write equations to solve for the same. There was boundary layer separation, complex wake flow, long trailing vortices which were getting generated at the rear but it was unable to solve for the same analytically and hence this was a grey area in aerodynamics. But with the advent of Computational Fluid Dynamics (CFD), the computers are capable of solving such equations and being very fast at the same time. Anderson Jr.[6] describes CFD as the art of using discretized algebraic forms in place of integrals or the partial derivatives, which in turn are solved to obtain numbers for the flow field values at discrete points in time/space. So the focus has again gradually shifted to passive flow devices which improve the drag and lift forces acting on the body. Various researchers $[3,7,8]$ have concluded that CFD is an approximation of the equations by averaging it with time. But more complex equations need to be solved to get more accurate results. As such CFD is now more of a research tool than a design tool and it can be calibrated with experimental data to ascertain its accuracy.

\section{DRAG REDUCTION EFFORTS}

\subsection{Ahmed Body:}

Ahmed et al. [9] designed a simple model for a car as shown in Fig-2 and this constituted of nose shaped front and a movable slant plane at the rear to conduct experiments at different rear angles to study the flow pattern. The nose and the slant plane was connected by a rectangular box. Wake separation flow behind the Ahmed body is the main contributor to the drag force, therefore its accurate prediction is important. Further experimental investigations included the effect of back light angles in the range of $0^{0}$ to $40^{\circ}$.

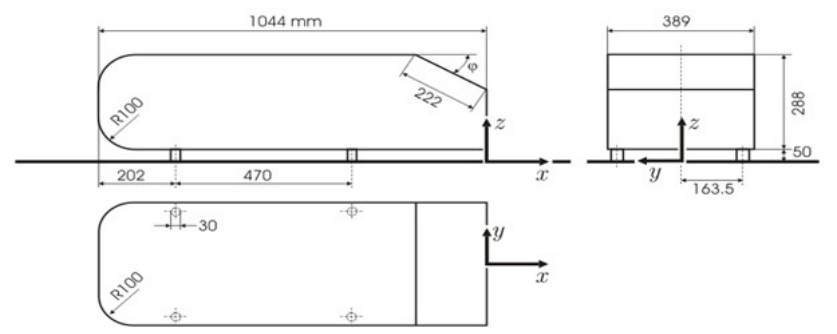

Fig-2: Geometry of the Ahmed Body [10]
They concluded that aerodynamic drag was very dependent on the rear slanted window and the critical angle was about $30^{\circ}$. For the angles lower than critical value, the flow separated at the roof of the vehicle. It reattached at the slant region and then separated again as it approached the rear. When the angle was near to $30^{\circ}$ the form drag increased drastically and then beyond the critical value of $\alpha$, the drag reduced again and was more or less constant till the maximum value of $\alpha$. Morel [12] observed that the $90 \%$ of the pressure drag force is contributed by slant surface and the rear base. The rear base alone accounts for more than $70 \%$ of the total drag force. The values recorded by Morel and Ahmed were graphically compared by Dumas [11] as shown in Fig-3.
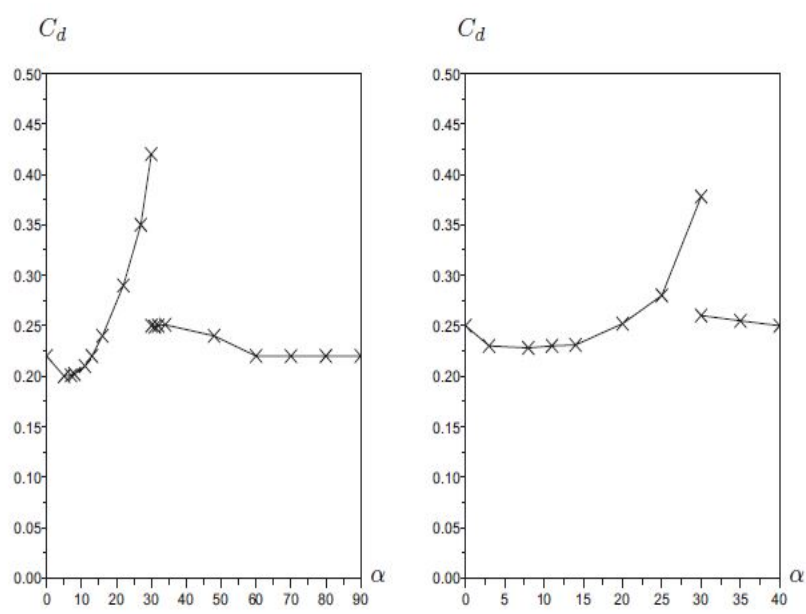

Fig-3: Drag measured for the Morel (left) and Ahmed (right) bluff-body for variousslant angles [11]

For a standard car, Table-1 displays the contribution of total drag over various elements of the car.It shows in particular that $70 \%$ of the drag coefficient depends on the external shape. Such a large value justifies the interest of a numerical modeling of the problem in order to find innovative external shapes that will largely reduce the drag coefficient.

Table-1:Drag Repartition on a realistic car [11]

\begin{tabular}{lc}
\hline Position & percent of $C_{d}$ \\
\hline Upper surface & $40 \%$ \\
Lower surface & $30 \%$ \\
Wheels & $15 \%$ \\
Cooling & $10 \%$ \\
Others & $5 \%$ \\
\hline
\end{tabular}

\subsection{Diffusers}

A diffuser is an aerodynamic device located on the underside of a road or racing car as depicted in Fig-4. It has to speed up the airflow entering the device (lowering pressure), and then slowing it down (increasing pressure) by means of the venture effect. While doing this it also has to make sure that flow separation does not occur, resulting in stalled and turbulent airflow-in other words a lot of drag. This is 
achieved by a gradual gradient and volumetric increase along the body of the diffuser. Since it has minimal drag penalties compared to other aerodynamic devices, it is very efficient for down force generation.

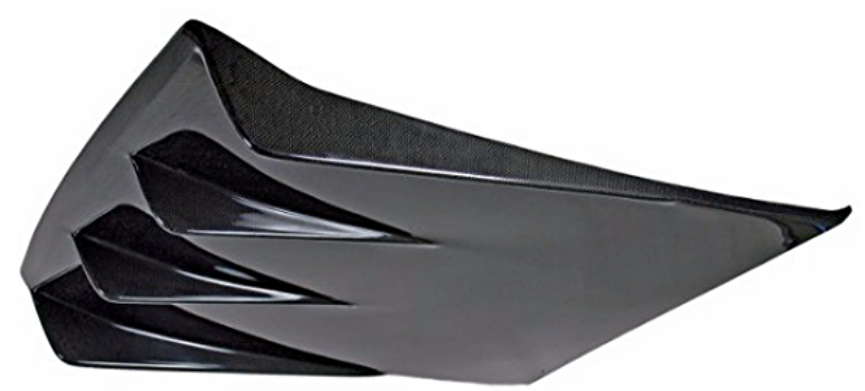

Fig-4:. A model of a diffuser used in race cars.

The first study investigating diffuser flows in a generic model was conducted by George [13] and George \& Donis [14]. They used a diffuser angle of $10^{\circ}$ over a range of $\mathrm{h} / \mathrm{L}$ values where $h$ is ground clearance and $L$ is vehicle's length. They found that downforce will increase with reduced clearance, aneffect that will peak when $\mathrm{h} / \mathrm{L}$ goes below 0.05 . Ruhrmann et al.[15] then studied the variations in downforce and drag coefficient over diffuser angles ranging from 5 to $20 \mathrm{deg}$ with moving ground and found that the diffuser over an angle of $14^{\circ}$ stalled and created less downforce. Ahmed et al.[16] in their diffuser at $3.45^{\circ}$ had to halve their original ground clearance to gain a lot of downforce which came to around 0.094 as it coefficient of lift. Marklund [17] tested on a sedan and wagon of a same manufacturer for a range of diffuser angles from 0 to $14^{\circ}$. $\mathrm{He}$ concluded that lift forces were significantly reduced and the reduction was larger in case of sedan. Optimum diffuser angle for sedan was $8^{\circ}$ and $5^{\circ}$ in case of wagon.

\subsection{Vortex Generator}

The main function of vortex generator is to delay the air flow separation. They are small and fit inside the boundary layer, shaped like triangular fins (See Fig-5) and are especially effective in speeds in excess of $100 \mathrm{kmph}$.

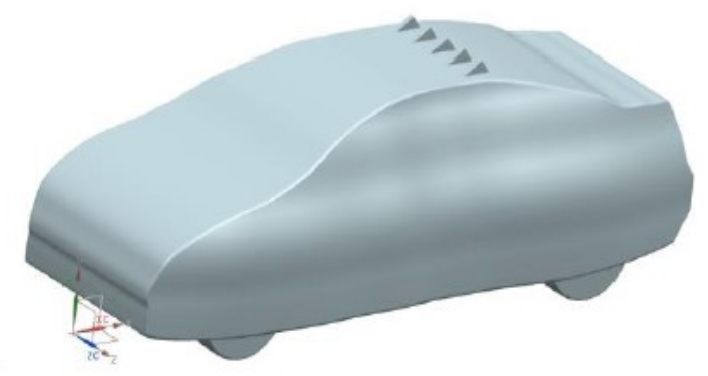

Fig-5: Sedan car model with vortex generator [8]

Katz [18] published a work regarding the incorporation of vortex generators onto the ends of the underbody of an Indycar. In it he graphically represented his data from Katz et al $[19,20]$ which shows that the lift of the car was very low at low ride heights about and the drag coefficient was reduced from 0.2 to 0.18 with addition of vortex generators.
Flow visualizations indicate that with reduced ground clearance the vortex strength increases and the vortices untangle and get closer to thevehicle's surface increasing suction force. Ahmed et al. [16] designed a vortex generator to be installed on the roof and it reduced the drag coefficient from 0.41 to 0.39 which shows its massive advantage at high speeds. Kumar et al. [21] designed vortex generators for sedan and hatchback and concluded with similar gains in drag coefficient of around 0.009 over conventional model.

\subsection{Reynolds Averaged Navier-Stokes Equation}

The Reynolds Averaged Navier-Stokes equations (RANS) are used to predict the fluid flow using a time averaged formulation. An instantaneous quantity is decomposed into its time averaged and fluctuating quantities. This concept is known as Reynolds decomposition It helps in simulating turbulent flows. Krajnovi et al. [22] conducted investigations on $25^{\circ} \mathrm{Ahmed}$ body using Large Eddy Simulations (LES) at low Reynolds number $\left(2 \times 10^{5}\right)$ and compared it with experimental data of Lienhart et al.[23] which showed that the LES was giving very satisfactory results. Kapadia et al. [24] also performed unsteady simulations on the model using the Re-Normalization Group (RNG) k- $\varepsilon$ turbulence model and concluded that it overpredicts the drag coefficient which was undesirable. This gave way to Lanfrit[25] to say with certainty that RANS was suitable for aerodynamic analyses over LES and DES inspite of them being superior in performance because of lesser simulation time needed in RANS.

\section{CONCLUSION}

It can be concluded after going through all the previous research carried out that CFD is still in its nascent stages and it cannot be completely relied upon for current design requirements. It needs to be used in tandem with experimental and numerical approaches so as to ascertain our calculations and to ideally predict the flow pattern and the losses being generated. But CFD has its own advantages in it being very easy to post process and in the long run much quicker to work with. Further research is needed for passive devices in commercial vehicles. There are still uncertainties surrounding the design and the dimensions are still unattributed to various models. Also work needs to be done on reducing the aerodynamic noises which might enhance the quality of ride for the passenger.

\section{REFERENCES}

[1]. W.H.Hucho, Aerodynamics of Road Vehicles, Butterworth-Heinemann, 1990.

[2]. P.N.Krishnani, (2009)"CFD study of drag reduction of a generic sports utility vehicle", Master's thesis, Mechanical Engineering Department, California State University, Sacramento.

[3]. Singh J., Randhawa J. (2014), "CFD Analysis of Aerodynamic Drag Reduction of Automobile Car-A Review", International Journal of Scientific Research vol3 Issue 6. 
[4]. M.M.Islam et al.(2010), “ Computational Drag Analysis over a car body", The International Conference on Marine Technology, Dhaka, Bangladesh.

[5]. M.Desai, S.A.Channiwala and H.J. Nagarsheth(2008), "A comparative assessment of two experimental methods for aerodynamic performance evaluation of a car", Journal of Scientific \& Industrial research, Vol.67, pp 518-522.

[6]. J.D.Anderson Jr., Computational Fluid Dynamics: The Basics with Applications, McGraw Hill Publication, 1995.

[7]. R.S.Khan, S.Umale(2014), "CFD Aerodynamic Analysis of Ahmed Body", International Journal of Engineering Trends and Technology (IJETT)-Volume 18 No7 Dec 2014, pp 301-308.

[8]. P.N.Selvaraju et al(2015), "Analysis of Drag and Lift Performance in Sedan car model using CFD", Proceeding of National conference on recent trends and developments in sustainable green technologies, pp 429-435.

[9]. Ahmed, G. Ramm and G.Faltin. (1984), "Some salient features of the time averaged ground vehicle wake", SAE Paper 840300.

[10]. S. Banga et al.(2015), "CFD Simulation of Flow Around External Vehicle: Ahmed Body", IOSR Journal of Mechanical and Civil Engineering Vol.12 Issue4, pp 87-94.

[11]. Laurent Dumas, "CFD-Based Optimization for Automotive Aerodynamics", University Pierre et Marie Curie, Paris, France.

[12]. Morel, T.: Aerodynamic drag of bluff body shapes characteristic of hatch-back cars.SAE Paper 7802670 (1978)

[13]. George AR. 1981. Aerodynamic effects of shape, camber, pitch, and ground proximityon idealized ground-vehicle bodies. ASME J. Fluids Eng. 103(12):631-38.

[14]. George AR, Donis JE. 1983. Flow patterns, pressures, and forces on the undersideof idealized ground effect vehicles. In Aerodynamics of Transportation II, ed. TMorel, J Miller, 7:69-79. New York: ASME.

[15]. Ruhrmann A, Zhang X. 2003. Influence of diffuser angle on a bluff body in groundeffect. J. Fluids Eng. $125: 332$

[16]. Ahmed.H.,Chacko.S. (2012), “Computational Optimization of Vehicle Aerodynamics", Proceedings of the 23rd International DAAAM Symposium, Volume 23, No.1, ISSN 2304-1382, pp 313-318.

[17]. Marklund.J., "Under-Body and Diffuser Flows of Passenger Vehicles" $\mathrm{PhD}$ Thesis in Machine and Vehicle Systems, Department of Applied Mechanics, Chalmers University of Technology, Gothenburg,Sweden (2013).

[18]. J.Katz," Aerodynamics of Race Cars", Annu. Rev. Fluid Mech.2006, 38:27-63, pp27-63.

[19]. Katz J, Garcia D. 2002. Aerodynamic effects of Indy car components. SAE J. PassengerCars: Mech. Syst., pp. 2322-30. SAE 2002-01-3311.
[20]. Katz J, Garcia D, Sluder R. 2004. Aerodynamics of race car liftoff. SAE 2004-01-3506.

[21]. M.J.Kumar et al(2013), "Effect of Vortex generatorson Aerodynamics of a car: CFD Analysis", International Journal of Innovations in Engineering and Technology, Vol2 Issue1 April 2013, pp 137-144.

[22]. Krajnovi'c, S., and Davidson, L. (2004). LargeEddySimulation of the Flow around Simplified Car Model.2004 SAE World Congress, SAE Paper No. 2004-01-0227,Detroit, USA, 2004.

[23]. H. Lienhart and S. Becker. (2003). Flow and turbulentstructure in the wake of a simplified car model. SAEPaper 2003-01-0656, 2003.

[24]. Kapadia, S., Roy, S. (2003). Detached EddySimulation over a reference Ahmed car model. AIAAPaper 20030857.

[25]. Lanfrit, M. (2005). Best practice guidelines forhandling Automotive External Aerodynamics withFLUENT. (Ver 1.2), Fluent Deutschland Gmbh. 